\title{
Supporting the Information Management of Control Activities in a CIM Shop Floor Environment *
}

\author{
G. Loria, I. Mazón, F. Rojas \\ Instituto de Investigaciones en Ingenieria, Universidad de Costa Rica, Costa Rica \\ email: gmoloria@cariari.ucr.ac.cr
}

\author{
H. Afsarmanesh, M. Wiedijk, L.O. Hertzberger \\ Computer Systems Department, University of Amsterdam, The Netherlands \\ email: hamideh@fwi.uva.nl
}

\begin{abstract}
A CIM shopfloor environment can be supported by a collection of different activities, such as design, manufacturing, marketing, etc. Every activity may consist of a number of subactivities where a subactivity can be performed and represented by several agents. The "agents" are described here as components that perform activities through executing their specific "tasks". So far, for DCS (Distributed Control Systems), manufacturers have resolved the "information management", "information sharing" and "control" problems, through purchasing all instruments and control equipments from the same supplier. In real applications however, both within the CAC activity (among its sub-activities) and in relations among the CAC activity and other CIM activities, the industrial processes define differently formatted data, for instance the discrete and continuous variables that need to be shared among several multivendor control equipments. Therefore, the information sharing and management and the control problems are still the difficult technological problems without an integral solution. This paper focuses on the Computer Aided Control (CAC) activity and describes some agents involved in process control and the tasks that they perform. Furthermore, an architecture is described to support the information management requirements for agents' cooperation in a CIM shopfloor environment. This information management architecture is then applied to the definition of cooperating CAC agents.
\end{abstract}

\section{Keywords}

Integrated CIM activities, Computer Aided Control activities, Federated Information Management system

\section{INTRODUCTION}

With the new trend of applying the concurrent engineering methodologies to product manufacturing (Barker, 1993; Afsarmanesh et al., 1994b), regular evaluation of activities involved in the "product life-cycle" increases the productivity, improves the quality of products, and reduces

\footnotetext{
*This research is partially supported by the CIMIS.net project ECLA 004:76102. 
the costs. Each activity in the product life-cycle, ranging from design and manufacturing to marketing and sales, involves a group of engineers and experts. Any improvements require that a part of the information is shared and exchanged within one activity (i.e. tightly linked) and with other activities (i.e. loosely linked) (Barker, 1993). The CAD, CAM, CAC, etc. activities support distinct phases of the product life cycle (Didic, 1993; Sepehri, 1987). Every CAx activity consists of several subactivities and executes specific tasks. A subactivity can either be executed by a single agent or in a cooperation of several agents which are, depending upon their tasks, either loosely or tightly linked. Typically, a task is supported by an agent. For instance, the CAC activity is represented by one or more agents that execute several control tasks, such as process control and quality control. Other activities associated with CIM, for instance: design, research and development, marketing, management, process and product planning, etc. can all be represented by their agents. Thus, an agent is a hardware-software basic cell that partially or totally performs one or more tasks within a (sub)activity. Quite often, these agents run on several independent and heterogeneous systems. The relationships among the CAD, CAM and CAC

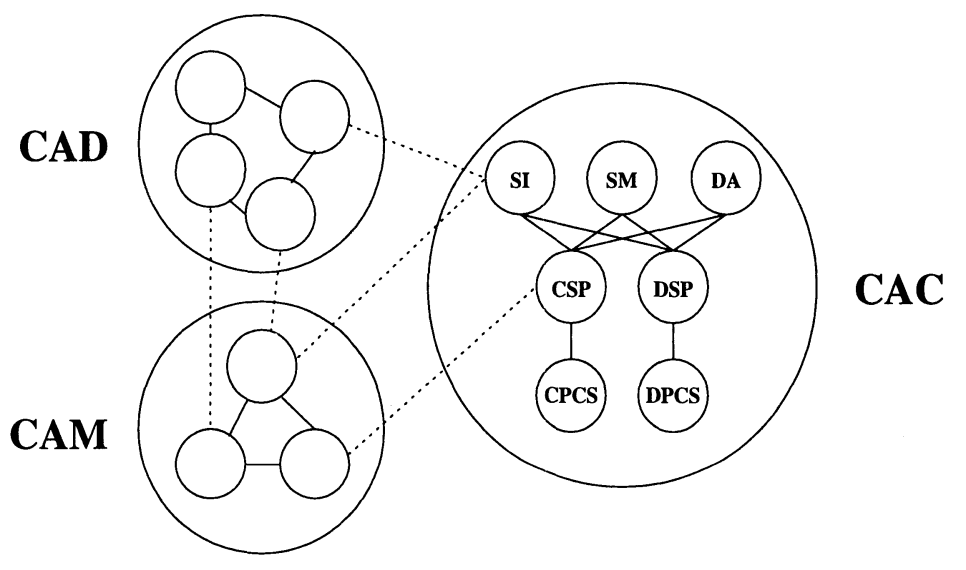

Figure 1 The tightly coupled CAC agents (represented by solid lines) and their loose coupling with CAD and CAM (represented by dotted lines).

activities, as illustrated in Figure 1, create an environment that supports the integration of the activities in the shop-floor. The CAD activity supports several subactivities, such as the geometrical design tasks and strategical control design tasks for CACSD (Computer-Aided Control System Design). CAD subactivities in turn perform certain tasks, for instance the geometrical modeling (design of parts, assemblies, etc.), simulation of control strategies (discrete and continuous), tuning (continuous process), and the timing and counting (discrete process). The CAM activity supports the supervision over geometrical product data and control of the numerical machines, FMS and the capacity evaluation of CAD parts. The main role of the CAC activity is to check and regulate the process variables on the shop floor, so that the processes have the adequate reliability and security. CAC performs some automatic tasks but mostly follows the operator supervisory recommendations in order to perform an optimal control over this important phase of the product life-cycle. The CAC activity mainly involves agents that perform control tasks such as continuous process control, discrete process control, the acquisition and monitoring of process variables, the display and management of alarms, communication services, optimization 


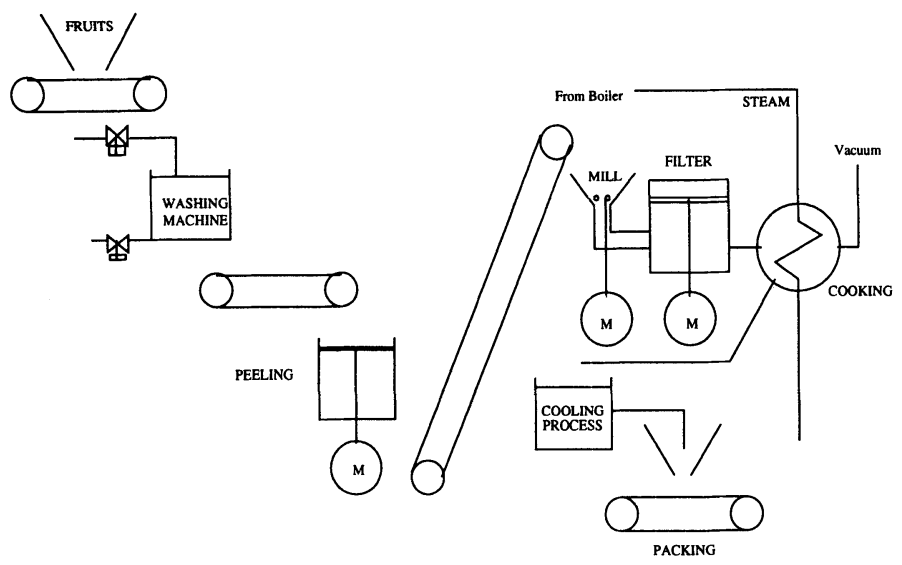

Figure 2 The marmalade production process

routines, supervision strategies, and contingency decision using expert systems. These tasks are described in more details in Section 2.

This paper discusses the requirements to achieve an integration of Shop-Floor CAC activities involved in CIM industry. Although, the focus of this paper on defining an information management architecture to support the CAC activity and the integration of its agents, this architecture is general enough to be applied to any other CAx shop floor activity. The remaining of this paper is organized as follows. Section 2 describes a marmalade application environment and the agents and tasks they perform in the continuous and discrete process control and supervision subactivities. Section 3 discusses the information management requirements for these activities, followed by a brief description of PEER's federated integration architecture in section 4 . Section 5 defines some example PEER agents for the CAC activity using the PEER integration architecture, followed by an example of the information sharing among these agents.

\section{A MARMALADE PRODUCTION APPLICATION}

In this section we first describe an application environment; a marmalade production industry. Figure 2 shows the marmalade production processes, that consist of interrelated discrete (batch), batch/continuous and continuous processes, and their interrelationships, similar to the description of continuous and discrete events in (Eigi M., 1993) and discrete events in (Walter, 1992; Eigi, 1990). The production begins with the cleaning and peeling of the fruits. The raw material is transported to a mill system. After filtering, the glucose content is measured and a special formula will be added. This product is then transferred to a tank for dehydration and cooking. This tank works at vacuum pressure with temperatures around $60^{\circ} \mathrm{C}$, the temperature is obtained by steam that comes from the boilers. The cooking process takes about 72 hours and is controlled by a specialist that knows the special aspects of the product. Once the cooking process has finished, the product is transferred to another tank. After the marmalade obtains ambient temperature the resulting product is packaged. The rest of this section describes the subactivities and agents related with the CAC activity in this marmalade industry and their interrelationships. 


$\begin{array}{lll}\text { Subactivity } & \text { Agent } & \text { Task } \\ \text { Continuous Process Control } & \begin{array}{l}\text { Continuous Process Control Strategies (CPCS) } \\ \text { Continuous Step Processor (CSP) }\end{array} & \begin{array}{l}\text { PID } \\ \text { Field Measurement } \\ \text { Communication }\end{array} \\ \text { Discrete Process Control } & \begin{array}{l}\text { Discrete Process Control Strategies (DPCS) } \\ \text { Discrete Step Processor (DSP) }\end{array} & \begin{array}{l}\text { Field Measurement } \\ \text { Communication }\end{array} \\ \text { Supervision } & \begin{array}{l}\text { Display agent (DA) } \\ \text { Signal Management (SM) } \\ \text { Signal Integrator (SI) }\end{array} & \end{array}$

Figure 3 Subactivities, agents and tasks in the CAC activity

Three CAC subactivities can be distinguished for the marmalade industry, namely continuous process control, discrete process control, and supervision. Figure 3 shows the main agents and tasks involved in each subactivity.

\subsection{Continuous process control subactivity}

The continuous process control subactivity involves the control of the specific hardware in the shop floor whose characteristic signals (input/output) are continuous. The main agents performing this subactivity are described below.

\section{Continuous Process Control Strategies agent (CPCS)}

The CPCS agent is responsible for the regulation of the variables of continuous processes using either local, remote, or other control setting parameters. In case of a local setting, the operation conditions are set by an operator directly on the controller hardware. However, this kind of setting is undesirable for this type of control environment. Usually, the control strategy can be changed at any time by a remote setting, through an agent in the CAC activity. CPCS usually runs on a dedicated computer and its most important task is the PID control task.

- The PID Control Task executes a special algorithm to control a continuous process in a feedback manner; using continuous variables from sensors (temperatures, pressure, flow, etc.), it calculates errors (regulation and tracking errors) and returns the command signals to process actuators (valves, motors, etc.). Usually, there are many continuous control loops associated to the control of this kind of shop floor environments.

\section{Continuous Step Processor agent (CSP)}

The CSP agent provides a bi-directional interface among the internal CAC subactivities, in order to communicate information of several shop floor equipments, and to transfer this information to other CIM activities. In the CIM environment, the CSP agent converts CAC activity information (such as field information) into a representation that can be used by other CAx activities; in the same way the information of the other CAx activities are translated into CAC representations. This agent translates the continuous variables to neutral files by means of STEP (Sastrón, 1993). Two tasks of this agent are described below: 
- The Field Measurement Task reads the signal values from different kinds of equipments such as sensors, transmitters, final elements and intelligence systems. These equipments require many proprietary interfaces and protocols to link them together. An implementation can be obtained using the field bus standard ISA SB50, that enables a real multivendor instrument integration at the shop-floor level.

- The Communication Task enables the communication between the process control with both the CAC subactivities and with other CIM activities, while hiding the specific and distinct hardware and protocol aspects of the process control equipment, avoiding the problems related to the combination of different hardware (Sepehri, 1987).

\subsection{Discrete process control subactivity}

This subactivity involves the control of the specific hardware in the shop floor whose input/output signals are discrete. The main agents are described below:

\section{Discrete Process Control Strategies agent (DPCS)}

The DPCS agent realizes the control over sequential processes. The local or remote operation is similar to the CPCS agent. This agent controls numerical machines using programmable controllers or similar hardware. The main operations of this agent are: timers, counters, step sequences, combinational functions. The DPCS agent is involved in step by step operations that depend on discrete conditions. Historically the CAx tools support these kind of operations.

\section{Discrete Step Processor agent (DSP)}

The DSP agent has the same function as in the continuous process control subactivity. In the discrete process control subactivity the DSP agent operates with bidirectional discrete variables from/to the shop floor equipment and other CIM activities. This agent performs tasks similar to the CSP agent as described below:

- The Field Measurement Task operates in similar way as in the continuous step processor agent. However, it works with bidirectional discrete variables from/to the shop floor equipment and other CIM activities.

- The Communication Task communicates the information flow (on/off signals, counters and timer values) of the process control to other hardware of the CAC and CIM activities, while hiding the specific and distinct protocol aspects of the process control equipment.

\subsection{Supervision subactivity}

The supervision subactivity is at the highest level of the CAC activity hierarchy. It supports the coordination between the different lower level CAC agents and the relationship with other CIM activities. This subactivity has an intelligent strategy analyzer that coordinates and evaluates the process requirements and the actual state of its different controllers and generates reports and displays information at mimics and other hardware. The supervisory agent handles alarm exceptions generated by the CAx activity that requires responses from the lower level CAC activities. Based on its internal model and strategies, this subactivity minimizes the negative effects on the entire production process. Supervision subactivity is defined by the following three agents: 


\section{Display Agent (DA)}

This agent presents a global overview of the process environment through a user friendly interactive graphical interface using active image techniques. It generates reports for different kinds of hardware.

\section{Signal Management agent (SM)}

The alarm agent is a traditional mimic panel for the acknowledgment of contingency conditions. This panel is built with visual and sonic indicators. The mimic panel can be emulated in the monitor using the alarm agent information.

\section{Signal Integrator agent (SI)}

The continuous and discrete control processor agents transmit information using different formats. Therefore, it is necessary to implement the integration and conversion of different information formats into a standard transportable information format.

\section{INFORMATION MANAGEMENT REQUIREMENTS}

There is a need to support the information sharing and exchange among different tasks in order to support the requirements for the cooperation and integration of different CIM activities. Although the focus of this paper is on the CAC activity, the cooperation and integration issues are similar for all CAx activities that will also be addressed here briefly.

The agents in the CAC activity frequently need to access the information about the state of the shopfloor. This information is managed by agents, so if an agent needs some information, it has to obtain it from other agents. Within the CAC activity, it is usually crucial for an agent to have fast (near real time) access to that information, even if it is managed by other agents, so the integration architecture must support it.

When applying a concurrent engineering approach to manufacturing, all CIM activities benefit from the information sharing and exchange both among different agents within one activity and among multiple activities. For instance, the CAD tools can then evaluate the production process and the control strategies. If a designer decides that the design is completed and ready to release, then the new models and/or parameters can be directly transmitted to the control units. The open architecture for Computer-Aided Control Engineering CACE (Barker, 1993) that includes a framework reference model, will improve the CAD capabilities and the information exchange with other CIM activities. The CAM activity can be extended to include support for the information exchange among engineers in the CAD, CAM and CAC activities. Using the time conditions and results set by the CAM and CAC activities, the design and evaluation of the control strategies in a CACE environment can be improved. A specific agent in the CAM activity can establish the link between the CACE design tasks and the actual results of the CAC activity. This agent analyzes the $C A D$ specifications and assigns the CAC-CAM references, such as strategies of process controllers and tuning for the $\mathrm{CAC}$ activities, and the numerical control machine instructions for CAM activities. CAM alarm conditions could be shared with the CAC activity that will benefit from the resolution of contingencies, using the expert and optimization systems in that activity. To support the information management for the CAC activity in a CIM shop floor environment the following requirements can be distinguished: 
- Integration and transformation of low level information generated and transmitted by low level control hardware.

- Sharing and exchange of information among agents within the CAC activity. The integration among these agents is usually tightly coupled.

- Sharing and exchange of information among the CAC activity and the other CIM activities such as CAD and CAM. The integration of these activities is usually loosely coupled.

In Section 4, the PEER federated object oriented database system will be described. PEER provides an integration architecture that supports the information management requirements described in this section. In Section 5.1, some example agents are defined by their PEER schemas. Finally, Section 5.2 presents how the PEER federated system supports the sharing and exchange of information among several agents within the CAC activity.

\section{PEER FEDERATED INTEGRATION ARCHITECTURE}

The PEER federated information management system has been designed to support the information management and information sharing and exchange in a network of both loosely and tightly coupled agents. The PEER information model is binary-based and object-oriented, in which every piece of information, both data and meta-data, are represented as objects. A PEER layer is defined for every agent that needs to share and exchange information with any other agent in the CIM environment. For example, two PEER agents in the CAC activity can be tightly coupled, while an agent in the CAD activity is loosely coupled to an agent in the CAC activity. Section 5 below describes the application of PEER to support the information management requirements of the marmalade production application in more detail.

Each PEER agent in the federation network can autonomously decide about the information that it manages locally, how it structures and represents its information, and which part of its local information it wishes to export to other agents. Each agent can import information that is exported by other agents and transform, derive and integrate (part of) the imported information to fit its interest and interpretation. PEER is a pure federated system, there is no single global schema defined on the information to be shared by all agents in the PEER network, and there is no global control. In the PEER layer of an agent, the information is structured and defined by several kinds of schemas. There is a local schema LOC (representing the information managed locally), several import schemas IMPi (representing the information shared by other agents, where integer $\mathrm{i}$ identifies a specific import schema), several export schemas EXPj (representing the information that this agent wishes to share with other agents, where integer $\mathrm{j}$ identifies a specific export schema), and an integrated schema INT (representing the integration of the local and the imported information for this agent). The powerful integration facility of PEER (Tuijnman and Afsarmanesh, 1993; Afsarmanesh et al., 1993; Afsarmanesh et al., 1994a), including the distributed schema management and the distributed query processing, makes the distribution of information and the heterogeneous information representations among different agents transparent to the user. The definition, integration and derivation of information in PEER are supported by a declarative specification using the PEER Schema Definition and Derivation Language SDDL (Afsarmanesh et al., 1993). The SDDL language supports the integration, derivation, interrelation, and transformation of types, attributes and relationships.

A prototype implementation of the PEER system is developed in the $\mathrm{Clanguage}$ and includes two user interface tools (Afsarmanesh et al., 1994a), a Schema Manipulation Tool (SMT) 
and a Database Browsing Tool (DBT). The PEER system has been successfully used in the integration of cooperating expert systems for the control of power distribution networks (in ESPRIT ARCHON project) and the development of a planning toolbox (in ESPRIT PLATO project). At present, PEER is being applied to the integration of biomedical information systems (in DIGIS project).

\section{INFORMATION MANAGEMENT OF THE CAC ACTIVITY}

In this section, we focus on the definition of PEER agents representing some CAC activities described earlier in Section 2. In Section 5.1 two example PEER agents are defined that perform certain CAC activities. In Section 5.2 an example of the information sharing among these agents is defined using the PEER integration architecture described in Section 4.

\subsection{Designing the PEER agents}

For each CAC agent a PEER layer must be defined to support its integration and information sharing with other agents. In the PEER layer, first the local information structures (the LOC schema) must be defined. The LOC schema is comprehensive enough to support the definition of the information that must be shared with other agents (through the EXP schemas). The EXP schemas are derived from the LOC schema and support the transfer of information among different agents in CAC subactivities and other CIM activities. For instance, to support the fact that the continuous process control subactivity receives information from the low level hardware instruments in different formats. The EXP schemas defined in the PEER network can be imported by any agent that needs to access and use their remote information.

The Step Processor Agents described in Section 2 are indispensable components of the CAC activity. These agents interface between different CAC subactivities. The main task of these agents is to transform different low-level data formats into a unique and generalized format using EXPRESS from STEP (ISO/DIS 10303) definitions. EXPRESS is an excellent language that supports the translation of production information to neutral files. Similar to PEER, the data model describing EXPRESS is also binary-based, which makes PEER a suitable candidate to represent EXPRESS datastructures and entities. Using the PEER federated architecture, different CAC agents can link to other CIM activities through the Step Processor agents. Since the CAC activity is a very important component of hybrid systems, the connection of this and other activities by a PEER federated network is a step towards the entire CIM shop floor environment integration and the support for Concurrent Engineering.

The remaining of this section presents the schemas for the CSP and DSP agents defined in Section 2. The schemas are defined using the SDDL language of PEER (Afsarmanesh et al., 1993), as described in section 4 . These schemas are necessary to support the sharing and exchange of information among CAC agents.

\section{CSP agent information}

Figure 4 shows the entities defined and exported by the Continuous Step Processor (CSP) agent. Engineering_units is a base entity, that allows the identification of instruments. The analog_element entity defines an analog variable and the direct_analog_read entity defines its specific measurement done by a direct measurement device. Export schema EXP5 in Figure 5 


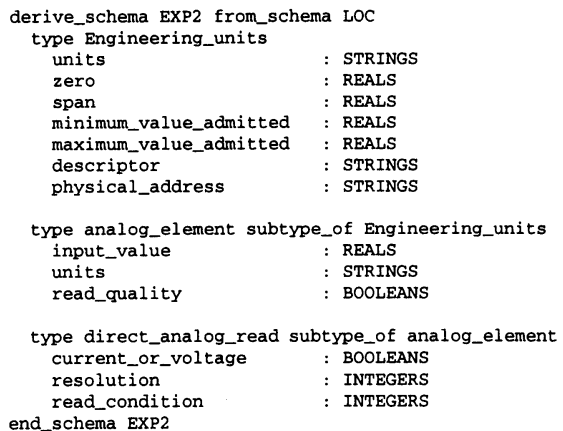

Figure 4 Export schema EXP2 of the Continuous Step Processor (CSP) agent

represents the global elements of the marmalade processing which are defined and exported by the CSP agent. The continuous_controller entity represents the variables and concepts of

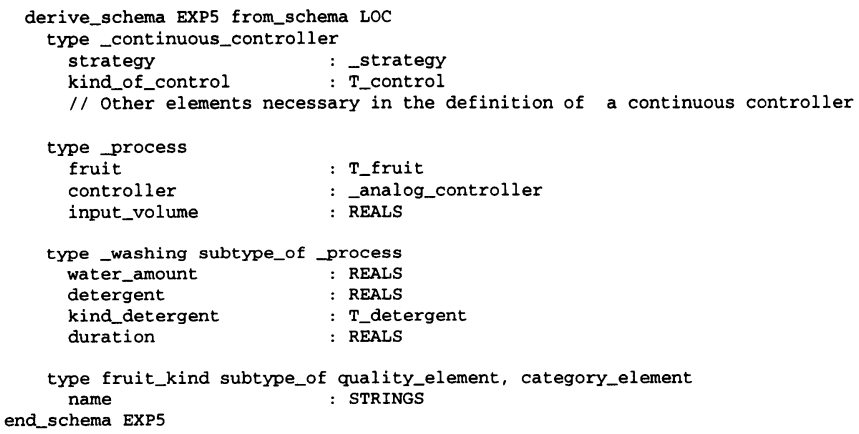

Figure 5 Export schema EXP5 of the Continuous Step Processor (CSP) agent

each controller type in the continuous step processor agent. The -process entity represents the process variables in the marmalade production industry. The _washing entity is a specialization of the _process entity and it represents the information specifically related to the fruit washing task. The fruit_kind entity defines a part of the essential physics conditions for the components involved in the marmalade industry. The quality_element and category_element are concepts associated with the particular conditions of the raw (fruit) material in the production process.

\section{DSP agent information}

Figure 6 shows the entities defined and exported by the Discrete Step Processor (DSP) agent. As the CSP agent, the DSP agent defines the Engineering_units entity, that allows the identification of instruments, as its base entity. The analog_element entity characterizes a digital variable, the 


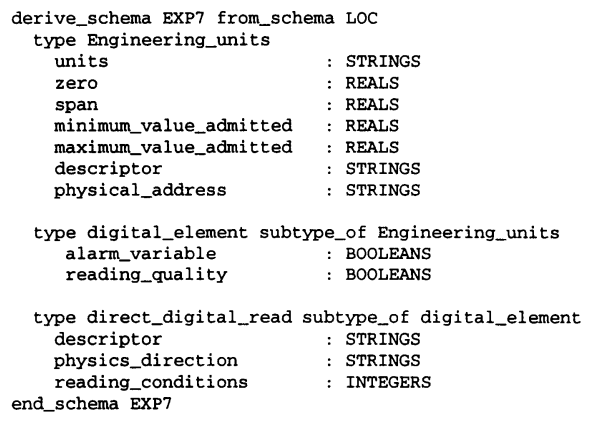

Figure 6 Export schema EXP7 of the Discrete Step Processor (DSP) agent

direct_analog_read entity defines the element value as a function of the physics meaning and the reading conditions.

\subsection{The integration approach}

An agent in the CAC activity frequently needs to access to information that is managed by other agents in the CAC activity. In this section we briefly describe an example of how the PEER architecture supports the integration of the field measurement information of the CSP and DSP agents by the Signal Integrator (SI) agent. This information includes the continuous process variables from sensors (temperature, pressure, flow etc.) transmitters, final elements, and intelligence systems, and the discrete process variables such as on/off signals, counters and timer values.

As a first step in the integration, the SI agent imports the export schema EXP2 of the CSP agent as its own import schema IMP4. The SI agent also imports the export schema EXP7 of the DSP agent as its own import schema IMP6. Then the second step for the SI agent is to create its own integrated schema (INT). The integration of the information of the CSP and DSP agents by the SI agent is defined in Figure 7 as the integrated schema of the SI agent. The first part in the INT schema represents the entities defined in the SI agent, then the second part represents the derivation specification of the SI entities in terms of the base entities as they are imported from the CSP and DSP agents. The SI agent defines its own view on the imported information and uses different names for some imported information. Namely, it interprets the Engineering_units entity as a process_variable, an analog_read as a continuous_variable, and a direct_read as a discrete_variable. The SI agent is not interested in the physical_address of an Engineering unit so that attribute is not a part of the process_variable entity. In the derivation specification, the union primitive defines the instances of the Engineering_units entities of both agents as the instances of the process_variable entity. The other entities are renamed as described above. The base entity names in the derivation specification are always extended with their import schema names to have a unique name to avoid confusion in case the entity names in different import schemas are synonyms. The integrated attributes of the process_variable entity are defined as the union of the values of the set of base attributes (enclosed by and ). For a complete description of PEER's derivation and integration primitives see (Afsarmanesh et al., 1993). 


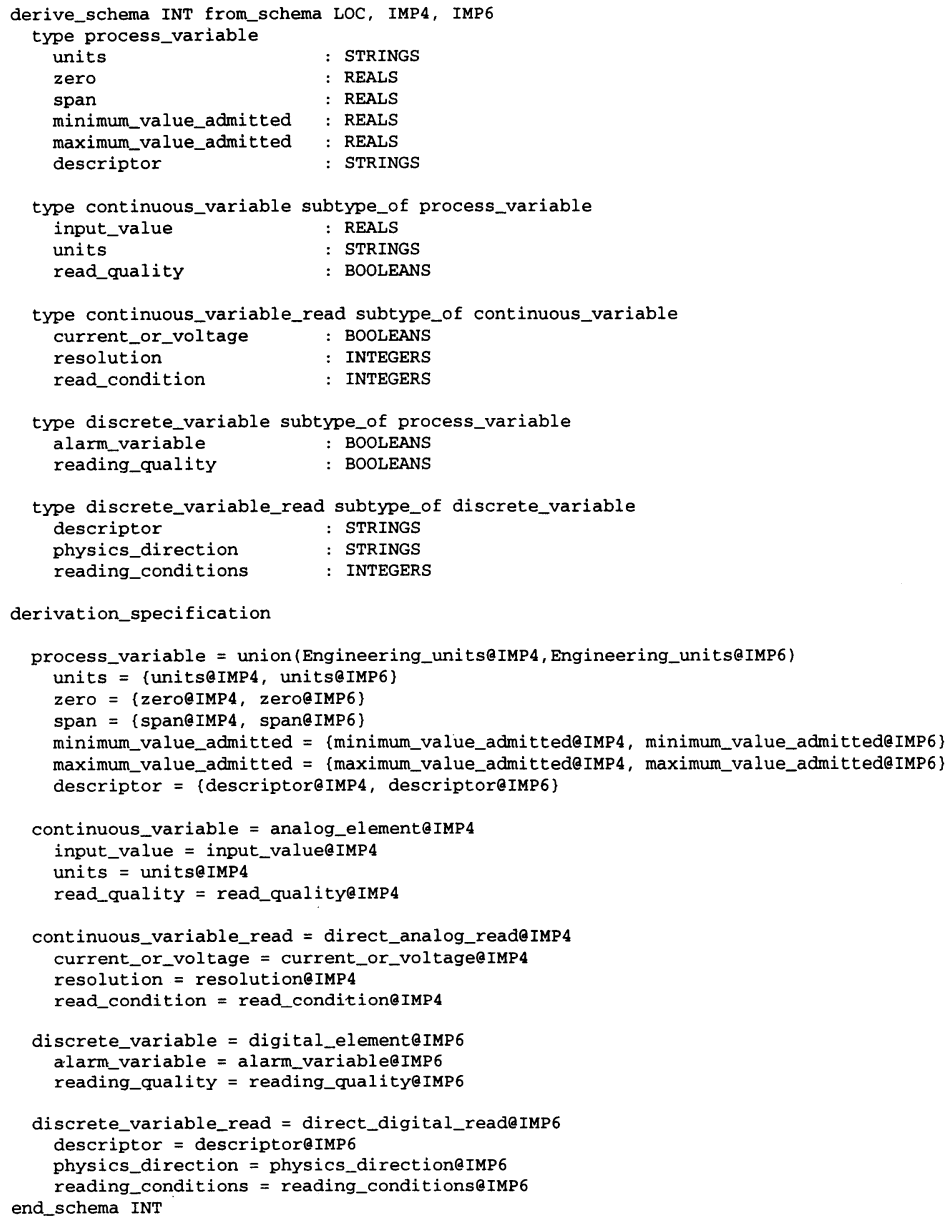

Figure 7 Integration of field measurement information by the Signal Integrator (SI) agent

The other agents in the supervision subactivity can be integrated in a similar way. The signal management (SM) agent needs access to the alarm information from the SI agent to acknowledge contingency conditions. The display agent (DA) needs the current state on the shop floor, for which it has to retrieve information from the step processor agents.

Similar to the methodology applied in Section 5.1, for every two agents that need to cooperate and exchange their information, we develop export schemas (EXPs) in the exporting agent. An export schema can be imported by the other interested agents as (one of) their import schemas (IMPs). The import schemas are then integrated with the local schema to generate the integrated 
schema (INT). Now the agent can use its integrated schema and transparently access any piece of information that is either managed locally or shared by other agents.

\section{CONCLUSIONS}

This paper first describes the CAC activities involved in a marmalade production industry. Each activity is represented by its agents that perform certain tasks. Then, an integration architecture, the PEER federated information management environment is described that supports the sharing and exchange of information among these agents. As an example, PEER is applied to the integration of the discrete and the continuous control activities on this shopfloor. Although the presented integration architecture is applied here mostly to Computer Aided Control (CAC) activities, the same architecture can support the information management of other multi-agent Computer Integrated Manufacturing environments.

\section{REFERENCES}

Afsarmanesh, H., Tuijnman, F., Wiedijk, M., and Hertzberger, L. (1993). Distributed Schema Management in a Cooperation Network of Autonomous Agents. In Proceedings of the 4th IEEE International Conference on "Database and Expert Systems Applications DEXA'93", Lecture Notes in Computer Science (LNCS) 720, pages 565-576. Springer Verlag.

Afsarmanesh, H., Wiedijk, M., and Hertzberger, L. (1994a). Flexible and Dynamic Integration of Multiple Information Bases. In Proceedings of the 5th IEEE International Conference on "Database and Expert Systems Applications DEXA'94", Athens, Greece, Lecture Notes in Computer Science (LNCS) 856, pages 744-753. Springer Verlag.

Afsarmanesh, H., Wiedijk, M., Moreira, N., and Ferreira, A. (1994b). Design of a Distributed Database for a Concurrent Engineering Environment. In Proceedings of the ECLA.CIM workshop, Florianopolis, Brazil, pages 35-43. (to appear in the "Journal of the Brazilian Society of Mechanical Sciences", ISSN 0100-7386).

Barker, A. (1993). Open Architecture for Computer-Aided Control Engineering. IEEE Control Systems, 13(2):17-27.

Didic, M. (1993). Voice: Synergy in Design and Integration. IEEE Software.

Eigi, M. (1990). Modelagem e Controle de Sistemas Produtivos: Aplicaçao da Teoria de Redes de Petri. Monografia no 55/90. Brasil.

Eigi M., P. (1993). Control de Sistemas a Eventos Discretos. Technical report, Sexto Escuela de Rob tica. CINVESTAV México.

Sastrón, F. (1993). STEP, una herramienta para el CIM. Automática e instrumentaciùn, (233).

Sepehri, M. (1987). Integrated Data Base for Computer-Integrated Manufacturing. IEEE Circuits and Devices Magazine.

Tuijnman, F. and Afsarmanesh, H. (1993). Management of shared data in federated cooperative PEER environment. International Journal of Intelligent and Cooperative Information Systems (IJICIS), 2(4):451-473.

Walter, A. (1992). Uso de las redes de Petri generalizadas para modelar sistemas dinámicos a eventos discretos. Revista Telegráfica Telefónica, (938). 